Western University

Scholarship@Western

Robarts Imaging Publications

Robarts Research Institute

$12-1-2013$

\title{
Image registration of ex-vivo MRI to sparsely sectioned histology of hippocampal and neocortical temporal lobe specimens.
}

Maged Goubran

Cathie Crukley

Sandrine de Ribaupierre

Terence M Peters

Ali R Khan

Follow this and additional works at: https://ir.lib.uwo.ca/robartspub

Part of the Bioimaging and Biomedical Optics Commons

Citation of this paper:

Goubran, Maged; Crukley, Cathie; de Ribaupierre, Sandrine; Peters, Terence M; and Khan, Ali R, "Image registration of ex-vivo MRI to sparsely sectioned histology of hippocampal and neocortical temporal lobe specimens." (2013). Robarts Imaging Publications. 13.

https://ir.lib.uwo.ca/robartspub/13 


\title{
Image registration of ex-vivo MRI to sparsely sectioned histology of hippocampal and neocortical temporal lobe specimens
}

\author{
Maged Goubran $^{\mathrm{a}, \mathrm{c}}$, Cathie Crukley $^{\mathrm{a}}$, Sandrine de Ribaupierre ${ }^{\mathrm{b}}$, Terence M. Peters ${ }^{\mathrm{a}, \mathrm{c}}$, Ali R. Khan ${ }^{\mathrm{a}, *}$ \\ ${ }^{a}$ Imaging Research Laboratories, Robarts Research Institute \\ ${ }^{b}$ Department of Clinical Neurological Sciences \\ ${ }^{c}$ Biomedical Engineering, Western University, London, Ontario, Canada
}

\begin{abstract}
Intractable or drug-resistant epilepsy occurs in to $30 \%$ of epilepsy patients, with many of these patients undergoing surgical excision of the affected brain region to achieve seizure control. Recent magnetic resonance imaging (MRI) sequences and analysis techniques have the potential to detect abnormalities not identified with diagnostic MRI protocols. Prospective studies involving pre-operative imaging and collection of surgically-resected tissue provide a unique opportunity for verification and tuning of these image analysis techniques, since direct comparison can be made against histopathology, and can lead to better prediction of surgical outcomes and potentially less invasive procedures. To carry out MRI and histology comparison, spatial correspondence between the MR images and the histology images must be found. Towards this goal, a novel pipeline is presented here for bringing ex-vivo MRI of surgically-resected temporal lobe specimens and digital histology into spatial correspondence. The sparsely-sectioned histology images represent a challenge for $3 \mathrm{D}$ reconstruction which we address with a combined $3 \mathrm{D}$ and $2 \mathrm{D}$ registration algorithm that alternates between slice-based and volume-based registration with the ex-vivo MRI. We evaluated our registration method on specimens resected from patients undergoing anterior temporal lobectomy $(N=7)$ and found our method to have a mean target registration error of $0.76 \pm 0.66$ and $0.98 \pm 0.60 \mathrm{~mm}$ for hippocampal and neocortical specimens respectively. This work allows for the spatially-local comparison of histology with post-operative MRI and paves the way for eventual correlation with pre-operative MRI image analysis techniques.
\end{abstract}

Keywords: Image Registration, MRI, Histology, Epilepsy, Anterior Temporal Lobectomy

\section{Introduction}

Intractable or drug-resistant epilepsy occurs in over $30 \%$ of epilepsy patients and is commonly charac3 terized by partial refractory seizures (Engel, 1998). When there is a localized focus, the standard of care

\footnotetext{
*Corresponding author

Email address: alik@robarts.ca (Ali R. Khan)
} 
4 for these patients is a surgical excision of the affected brain region in order to achieve seizure control (Engel 5 et al., 1992). Current clinical imaging protocols and surface EEG techniques of epileptogenic focus localization may not be sufficient for pre-operative planning due to limited sensitivity to deeper brain structures, and low resolution of source localization techniques (Smith, 2005). These limitations often necessitate invasive electrophysiological monitoring using sub-dural strips or grid electrodes. Magnetic resonance imaging (MRI) techniques such as diffusion tensor imaging (DTI), relaxometry mapping, high resolution functional o MRI (fMRI), voxel-based morphometry, and cortical thickness analysis can detect abnormalities not identi1 fied with conventional or diagnostic MRI protocols (Bernasconi et al., 2000, 2004; Bernhardt et al., 2009). This is important since it has been shown that post-operative outcomes can be predicted more accurately in 3 patients where lesions can be identified (de Tisi et al., 2011; Fish et al., 1993). These techniques also have 4 the potential to improve pre-operative localization of the focus, paving the way towards less invasive proce5 dures and better surgical outcomes. With the plethora of MRI sequences and analysis techniques available, 16 the challenge then becomes how to verify and evaluate the sensitivity and specificity of these techniques 7 for detection of abnormal brain tissue. Prospective studies involving pre-operative imaging and collection 1 of resected tissue from surgery (Eriksson et al., 2007; Howe et al., 2010) provide a unique opportunity for 9 verification and tuning of these image analysis techniques, since direct comparison can be made against high-resolution ex-vivo imagery, histology and immunohistochemistry. In order to carry out this verifica2 tion, spatial correspondence between the MR images and the tissue-derived histology images must be found, which remains challenging due to the deformations involved during resection of the tissue and histological processing and the difficulty of accurately registering the histology slices to the MR images. Registration is the task of finding this spatial correspondence by modelling the transformations and deformations that 25 occur due to surgical resection and tissue handling. These deformations can be divided into two types: 6 those occurring during surgical resection, and those occurring during histological processing. By obtaining 27 an intermediate ex-vivo MRI of the tissue specimen after surgical resection, we can divide this challenging registration problem into two independent sub-problems that can be tackled individually. This manuscript presents methodology and validation for the latter sub-problem, that is, the registration of ex-vivo MRI with histological slides to account for transformations due to slicing, slide mounting, and other histological processing. The proposed method, based on an iterative $3 \mathrm{D}$ and $2 \mathrm{D}$ image registration scheme, does not 32 require implanted landmarks, block-face images, or serially sectioned histology images, and achieves a target registration error of $0.76 \mathrm{~mm}$ and $0.98 \mathrm{~mm}$ for hippocampal and neocortical specimens respectively.

Histological verification of MRI is rare since tissue from surgeries or postmortem specimens are required, 5 and when available validation is not a straightforward task. A previous method has been devised to manually 36 match histopathology of temporal lobe resections to MRI using a cutting cradle to resample the MRI to the 37 orientation of histology (Eriksson et al., 2005). However no image registration was performed and only visual 38 assessment of the correspondence between the MRI and photographs of histology slices was accomplished. 


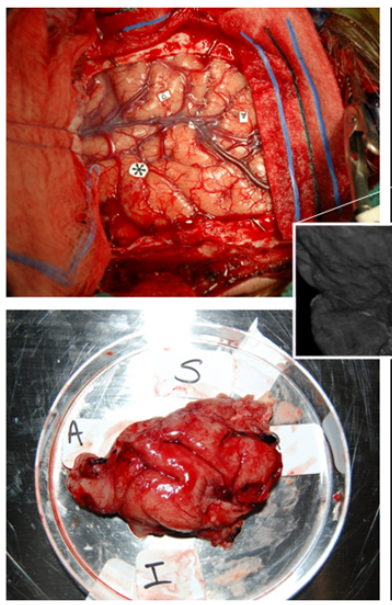

d) b)

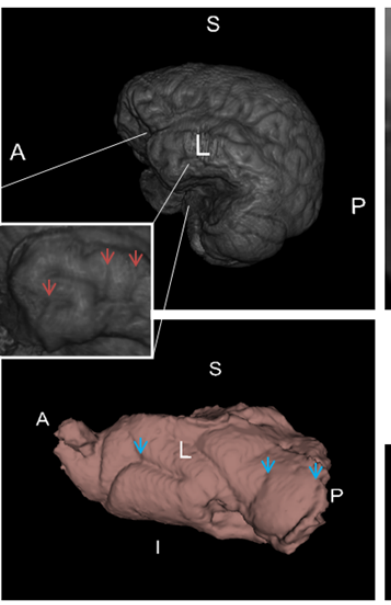

e) c)

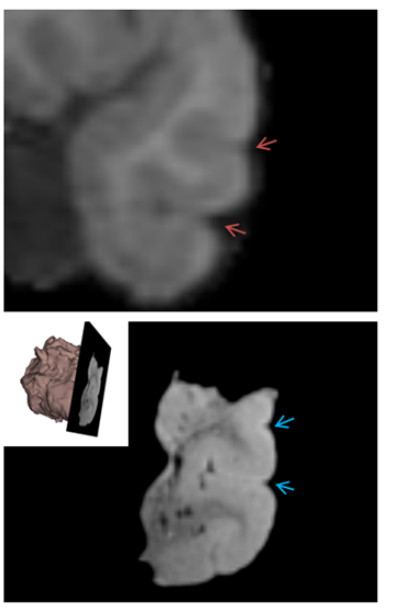

f)

Figure 1: Example of encountered deformations. Red arrows represent cortical sulci on the pre-operative MRI and blue arrows represent the corresponding sulci on the resected neocortex specimen. a) Photograph of surgical view before resection, b) Volume rendering of a pre-operative MRI of the patient with a zoomed view showing the temporal pole, c) Coronal view of the pre-operative MRI demonstrating temporal gyri with red arrows, d) Photograph of the temporal lobe neocortex post resection, e) Surface rendering of the resected neocortex with blue arrows showing the corresponding sulci to part b), and f) Coronal view of the ex-vivo MRI with blue arrows indicating sulci corresponding to part c); the small window in the top left corner demonstrates the mesial (interior) side of the neocortex with an intersection of the shown MRI slice. 
To evaluate the ability of high resolution MRI to resolve underlying pathologies of focal epilepsy an accurate full image registration is needed as the extent of pathologies such as focal cortical dysplasia (FCD), gliosis or hippocampal subfield sclerosis could be on the order of millimeters. Several challenges are met in the process of finding spatial correspondence, or registration, between resected tissue and preoperative MRI. One of the main challenges encountered is tissue deformation introduced due to the physical stresses experienced during surgery, as well as distortions to the tissue during the histological processing. Figure 1 demonstrates such tissue deformations encountered during a procedure. These deformations can be divided into primary and secondary categories (Dauguet et al., 2007). Primary deformations can be thought of as three dimensional changes, such as mechanical distortions during brain extraction once the resected specimen is detached from surrounding brain tissue, when cutting the specimen in blocks, or non uniform shrinkage induced by formalin fixation. Secondary deformations are within-slice distortions which are due to stretching of microtome cut sections on a water bath, spreading histology slices over glass slides and staining. Histology slices breakage, is a major manifestation of the deformations encountered during histological processing of the tissue. Furthermore, differential shrinkage of the tissue is another challenge that is due to the different intrinsic properties of white and grey matter. Registration of histology from surgically resected brain specimen to MRI is more challenging than registration of post-mortem or animal tissue as the tissue has to be sparsely sectioned, in comparison to the possibility of serially sectioning the entire specimen. This is limitation is imposed by the clinical requirement of pathology departments to keep parts of the resected specimen in tissue banks. The very different anatomy between sparsely sectioned adjacent histology slices (several $\mathrm{mm}$ apart) presents itself as another significant challenge. To address the challenge of non-rigidly registration $2 \mathrm{D}$ sparsely sectioned histology slides of brain resections from epilepsy surgery to in-vivo 3D MRI, we propose a full image registration protocol that relies on ex-vivo imaging of the specimen, to enable accurate correlation of histopathology with MRI. This work focuses on the intermediate registration of histology images to ex vivo imaging of hippocamapal and temporal lobe resections from anterior temporal lobectomies (ATL). Our protocol reduces the complexity of the in-vivo MRI to histology registration problem by leaving a single-modality $3 \mathrm{D}$ ex-vivo to in-vivo registration as the last step.

Presently, there are no automated histology to MRI image registration protocols that could be widely applicable to focal resections of human brain, such as tissue resected during epilepsy surgery. We present here a protocol to register ex-vivo scans of hippocampal and neocortical temporal lobe resections to histology as an intermediate step that reduces the complexity of the preopeartive MRI to 2D sparse histology problem. Specifically we describe a novel landmark-free algorithm for simultaneous reconstruction and alignment of sparsely sectioned histological data to ex-vivo MRI, and a quantitative validation for our registration method. Performing this intermediate step addresses most of the challenges of registration to in-vivo imaging due to the higher resolution and reduced deformations of the ex-vivo images. Furthermore, the higher resolution of specimen imaging is advantageous for examining the correlation between MRI and histology. The proposed 
method represents a significant step towards in-vivo MRI to histology registration in the clinical setting and can be broadly applicable to MRI and histolopathology correlations of resections other than epilepsy surgery.

\section{Methods \& Materials}

\subsection{Recruitment, surgery $\&$ specimen acquisition}

Seven patients suffering from intractable temporal lobe epilepsy (TLE) were recruited as part of an ongoing study. This project has been approved by the office of research and ethics of the University of Western Ontario, and informed consent was obtained from all patients prior to their recruitment in the study. All such patients were recommended for ATL surgery by the department of clinical neurological sciences at the University Hospital (UH) of the London Health Sciences Centre, and had preoperative investigations including neuropsychological testing and 1.5T clinical MRI scans which included T1w, T2w, FLAIR, and diffusion-weighted sequences. Patients were monitored with scalp-based electroencephalogram (EEG) video telemetry for seizure characterization, with three patients having to undergo monitoring with subdural placement of strip electrodes. In addition to the $1.5 \mathrm{~T}$ clinical MRI scans performed at the hospital, patients underwent a series of scans on 3T and 7T MRI research scanners, including high-resolution structural imaging, diffusion-tensor imaging, relaxation mapping and resting-state functional imaging prior to surgery. Following surgery, the resected tissue specimens were transferred to the Robarts Research Institute for ex-vivo specimen imaging on the same $3 \mathrm{~T}$ scanner and then to the pathology technologist for histological processing. From the seven patients, fourteen resected specimens were collected, but only twelve out of the fourteen were used in the study due to a fragmented hippocampus specimen and missed ex-vivo hippcampus scan. Table 1 summarizes the patients demographic data, as well as, their clinical MRI and histopathological findings.

\subsection{Specimen Ex-vivo MR Imaging}

After resection, each specimen was placed in a large petri dish within a specialized sealed cooler for specimen transport, and orientation labels were marked on the container by the operating neurosurgeon, with photographs taken for future reference. MR imaging was carried out on the specimens in two sessions: immediately following surgical resection, and after overnight fixation in 10\% formalin. For the initial session, referred to as the pre-fixation session, the specimens were immediately transferred from the operating room to the scanning suite at the Robarts Research Institute and prepared for imaging. Each specimen was wrapped in gauze for stabilization, transferred to suitably-sized containers for imaging, and immersed in a fluorine-based lubricant 'Christo-lube' (Lubrication Technology, Inc) prior to imaging to avoid susceptibility artifacts at the tissue boundaries. Identical preparation was performed for the second post-fixation session. 


\begin{tabular}{cccccccc}
\hline \hline Patient & Gender & Age & Onset age & Seizure origin & MRI & Path. & Scan Protocol \\
\hline 1 & F & 51 & 10 & Right & Normal & dysplasia & I \\
2 & F & 22 & 15 & Right & Normal & mild MTS & I \\
3 & F & 52 & 12 & Left & Non-specific & mild FCD & I \\
$4^{\star}$ & F & 26 & 20 & Right & Tuberous Sclerosis & Cortical tubers & II \\
5 & F & 22 & 15 & Right & R. MTS & MTS & II \\
6 & M & 20 & 3 & Left & L. MTS & MTS & II \\
$7^{\star}$ & M & 19 & 5 & Right & Normal & Gliosis & II \\
\hline \hline
\end{tabular}

Table 1: Summary of demographics and clinical data, including MRI and histopathological findigds, for the seven recruited patients in the study. Registration was performed on both hippocampus and neocortex specimens for all patients. In two cases (denoted by $\star$ ) registration was only performed on the neocortex due to a missed scan and a fragmented hippocampus specimen. MTS: Mesial Temporal Sclerosis. FCD : focal cortical dysplasia.

Specimen imaging was performed on a 3T Discovery MR750 scanner (GE Medical Systems, Milwaukee, WI, U.S.A.). Initially, an in-house developed gradient-insert coil was employed in the scanning setup with each specimen imaged sequentially using different coils. For improved time-efficiency in scanning and setup, the gradient-insert coil was not employed in later studies, and both specimens were imaged in the same field of view. Post-fixation T2-weighted scans were used in the subsequent image processing and registration. Both scanning protocols are described in detail below:

\subsubsection{Scan Protocol I}

The first protocol utilized a gradient-insert with a 4 channel TORO coil for the neocortex and a solenoid coil for the hippocampus, with each specimen scanned sequentially. T2-weighted images with a multiphase balanced SSFP FIESTA sequence with 4 cycled phases were acquired for the neocortex ( $\mathrm{TR}=3.5 \mathrm{~ms}$, $\mathrm{TE}=1.75 \mathrm{~ms}$, flip angle $=40^{\circ}, \mathrm{N}=4$, matrix $=200 \times 200$, slice thickness $\left.=0.3, \mathrm{FOV}=60 \mathrm{~mm}\right)$ and the hippocampus $\left(\mathrm{TR}=3.97 \mathrm{~ms}, \mathrm{TE}=1.98 \mathrm{~ms}\right.$, flip angle $=40^{\circ}, \mathrm{N}=4$, matrix $=200 \times 200$, slice thickness $\left.=0.3, \mathrm{FOV}=60 \mathrm{~mm}\right)$.

\subsection{Scan Protocol II}

For the second protocol, a 6 channel coil, designed to image the carotid artery, was used instead of the gradient-insert coil of the previous configuration. Similar T2-weighted FIESTA images (TR=8.17ms, $\mathrm{TE}=4.08 \mathrm{~ms}$, flip angle $=40^{\circ}, \mathrm{N}=2$, matrix $=200 \times 200$, slice thickness $=0.4, \mathrm{FOV}=120 \mathrm{~mm}$ ) with a resolution of $0.35 \times 0.35 \times 0.4 \mathrm{~mm}$, as well as, Fast gradient echo (fastGRE) scans with sixteen echoes $(\mathrm{TR}=65.0 \mathrm{~ms}$, 
$\mathrm{TE}=38.9 \mathrm{~ms}$, flip angle $=40^{\circ}$, matrix $=200 \times 200$, slice thickness $\left.=0.4, \mathrm{FOV}=120 \mathrm{~mm}\right)$ were acquired for the study. A switch was made to the second protocol due to the significant time savings achieved during the setup and gradient shimming processes (from $\sim 45 \mathrm{~min}$ to $\sim 5 \mathrm{~min}$ ) and minimal loss of image resolution.

\subsection{Histological processing}

Following pre-fixation and post-fixation MRI imaging, the specimens underwent accessioning and grossing at the Department of Pathology at UH, and were then cut into two halves midway, anterior-posterior, through the specimen. Each half of the specimen was then embedded in agar for a stabilization effect during slicing. The half-specimens were then sectioned parallel to the initial cut, into $4.4 \mathrm{~mm}$ pieces in the anterior to posterior direction using a deli slicer (Globe Food Equipment Company, Dayton, OH, U.S.A). Each block was embedded in paraffin and mounted on a microtome where $8 \mu \mathrm{m}$ thick sections were cut from the face of each block and mounted on slides. One slide from each block was stained with hematoxylin and eosin (H\&E) according to standard clinical neuropathology protocols, and additional stains or immunohistochemistry, including glial fibrillary acidic protein (GFAP), neuronal nuclei (NeuN) and neurofilament (NF), were ordered where deemed necessary by the neuropathologist on duty. The resulting slides were digitized on a ScanScope GL (Aperio Technologies, Vista, CA, USA) bright field slide scanning system at a maximum of 20x optical zoom, and stitched to form full-frame multi-resolution images stored in BigTIFF file format (maximum pixel resolution $0.5 \mu \mathrm{m})$. Since each specimen was sectioned into blocks of $4.4 \mathrm{~mm}$ thickness, the corresponding H\&E stained images have a physical spacing of effectively $4.4 \mathrm{~mm}$ in the coronal (anterior-posterior) direction. Figure 2 shows an overview of all these histological processing steps.

\subsection{Image registration}

To motivate our registration approach we first describe how the numerous physical processing steps between ex-vivo MR imaging and slide digitization affect the specimen, and how these steps could be accounted for with registration. As outlined in the previous section, after imaging, the tissue specimen is sectioned coronally, but this slicing plane is not enforced to be along the orthogonal axes corresponding to the MRI coordinate system. We therefore need to obtain a transformation between the MRI axes and tissue slicing axes. Next, when the specimen is mounted on the microtome, there may be variability in the angle at which sections are taken and in the number of partial sections removed before a full section is retained. This effectively leads to variability in the angle and spacing between sections. For similar procedures carried out on prostate specimens, Gibson et al. (2012) quantified the variability to be $1.7 \pm 1.1^{\circ}$ and $1.0 \pm 0.5 \mathrm{~mm}$ in angle and spacing respectively. Because of the relatively small magnitude of variability, and ease of working with parallel sections, we do not explicitly account for this and and instead assume sections are parallel and spaced by $4.4 \mathrm{~mm}$. The tissue being sectioned in the microtome is highly folded after the blade is brought down, thus to mount the section on a slide, it is first placed in a water bath to unfold, then eased onto 


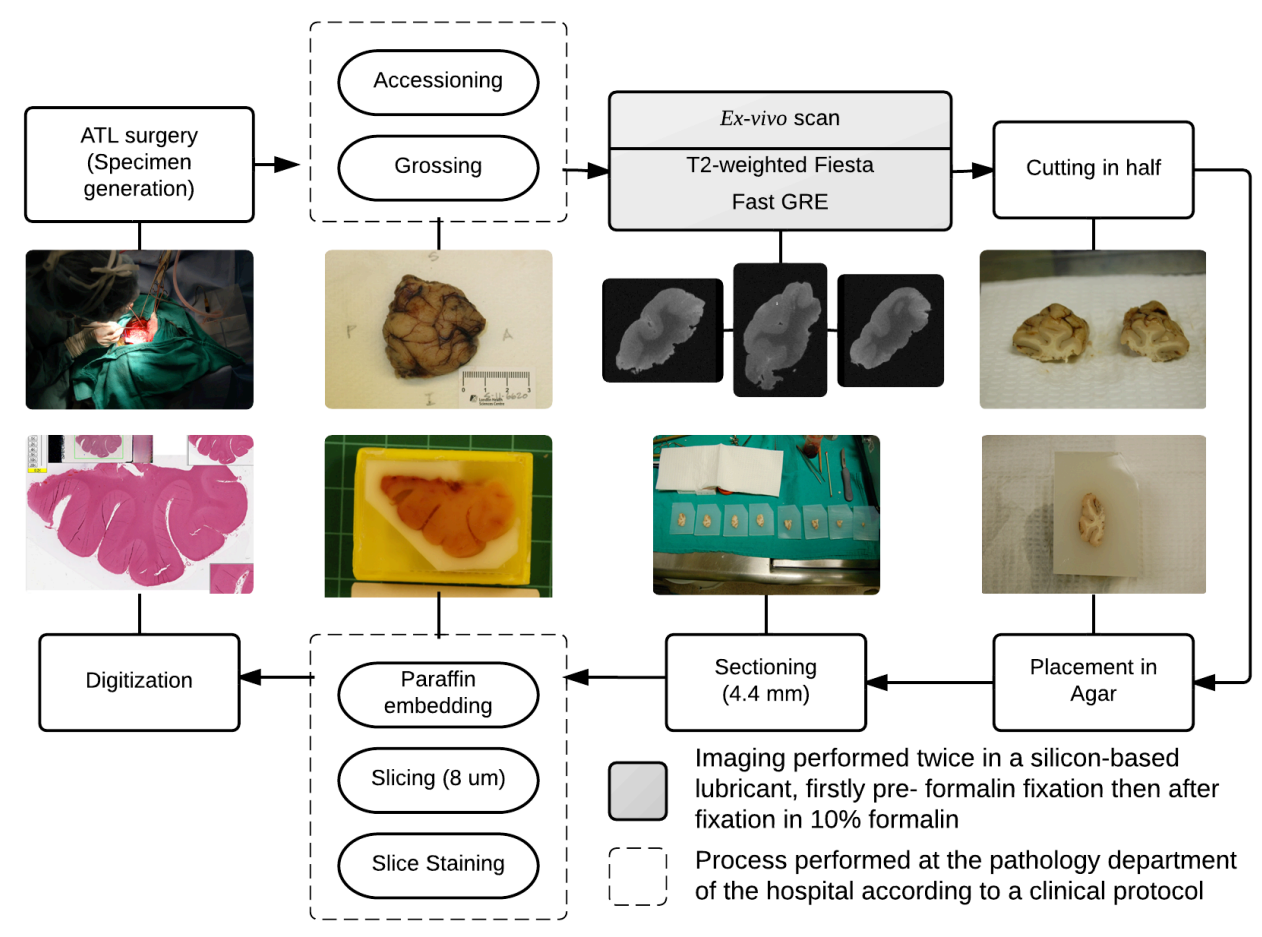

Figure 2: Overview of histological processing from specimen generation to digitization. Processed performed at in a standard clinical workflow at the hospital are included in dashed boxes. 
the glass slide. This procedure can introduce folds or tears in the mounted section, and placement on the slide is variable. Histological processing and staining of the section can introduce further distortions, such as differential shrinkage or expansion of tissue. Since all these deformations are present in the thin $(8 \mu \mathrm{m})$ section of tissue mounted on the slide, these can be modelled as transformations and warps constrained to the $2 \mathrm{D}$ plane.

In summary, we require a registration approach that can model: 1) the transformation between the 3D MRI axes to the specimen slicing axes (3D rigid transformation), and 2) the transformations and deformations of each slide-mounted section constrained to the $2 \mathrm{D}$ space of the slide (2D rigid transformations and non-rigid deformations).

\subsection{Iterative registration algorithm}

In this section we outline our iterative registration approach to attain the transformations and deformations and to establish correspondence between the MRI and histology images. Note that the registration procedures for hippocampus and neocortex images were carried out separately in each case. Preliminary results for neocortex registration were shown in Goubran et al. (2012).

If a $3 \mathrm{D}$ reconstruction of the histology were given, 3D rigid image registration could be used to align the MRI to the histology. However, to generate a 3D reconstruction of the histology, the individual histology slices would need to be corrected, using the registered MRI as a reference. Thus we see that obtaining the $3 \mathrm{D}$ rigid transformation is dependent on having a $3 \mathrm{D}$ histology reconstruction, and this is in turn dependent on the 2D histology registration with the MRI for a reference. To resolve this circularity, we propose an iterative registration scheme that alternates between 1) finding the 3D rigid transformations given the current histology reconstruction, and 2) finding the 2D rigid transformations and non-rigid deformations to reconstruct the histology volume given the current 3D rigid transformations.

Figure 3 presents a block diagram overview of our overall registration algorithm. First, the histology and MRI images are pre-processed separately to obtain image pairs of the same resolution and field of view suitable for image registration. Then the images are fed into an iterative registration algorithm that alternates between registration of the MRI volume to the current estimate of the histology volume (3D Rigid Registration), and registration of the histology slides to the reference MRI slides for histology volume reconstruction (2D Rigid Registration and 2D Non-rigid Registration). The details of this registration are shown in Algorithm 1.

\subsubsection{MR image pre-processing}

Prior to image registration, the images underwent a series of pre-processing steps, carried out with command-line tools from the FSL image analysis suite (FSL, http://fsl.fmrib.ox.ac.uk) and scripts written in MATLAB (The MathWorks Inc., Natick, MA, USA). First, ex-vivo MRI images scanned with 
Input: Histology and MRI volumes: $\mathbf{H}^{0}=\left\{H_{j}\right\}_{j=1 \ldots N}, \mathbf{M}$

Output: Final volume and transformations: $\mathbf{H}^{i_{\max }}, T_{3 D}^{i_{\max }}, T_{2 D, j=1 \ldots N}^{i_{\max }}, \Phi_{2 D, j=1 \ldots N}^{i_{\max }}$

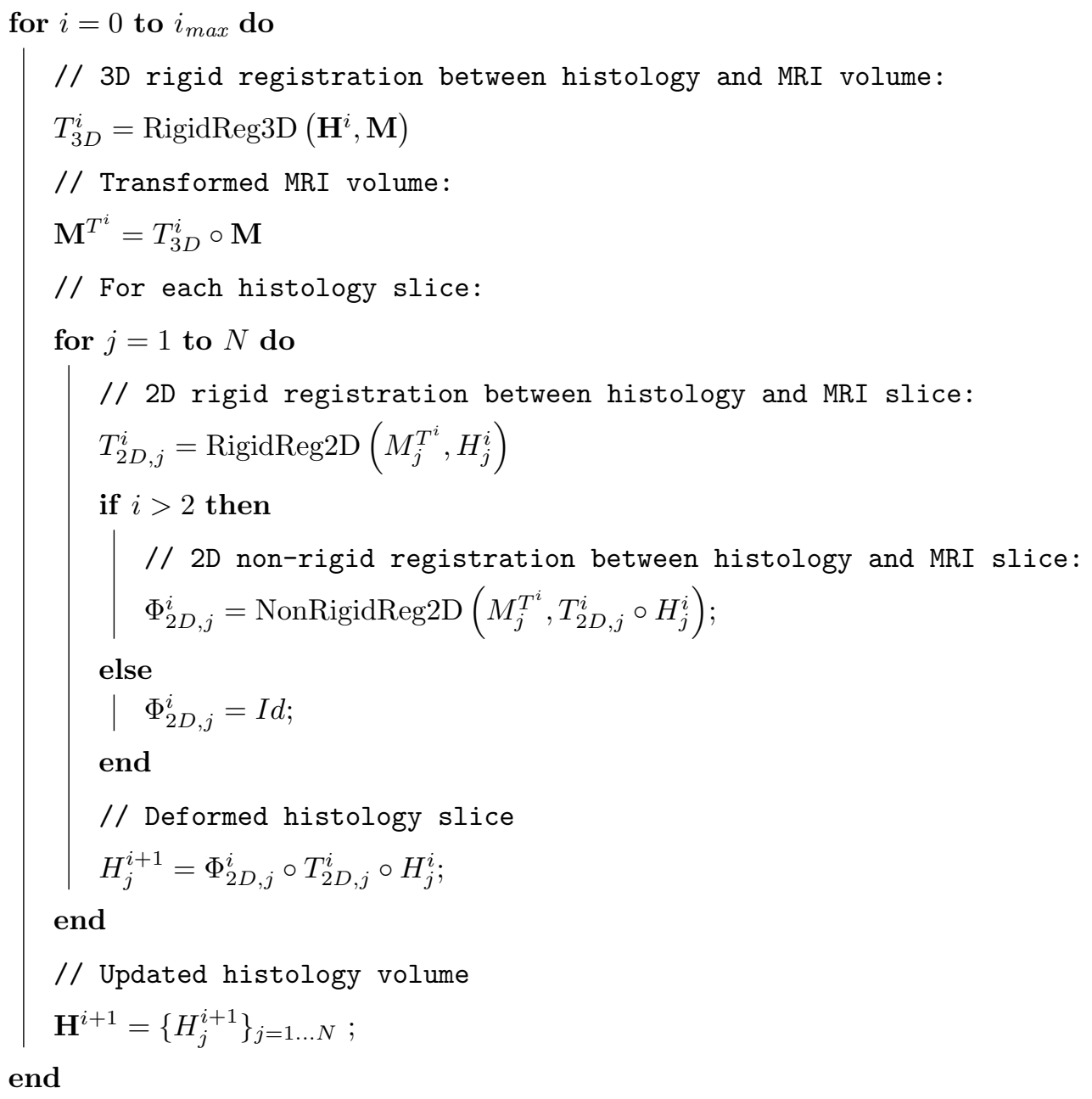

Algorithm 1: Iterative 3D and 2D registration of input histology volume $\mathbf{H}^{0}=\left\{H_{j}\right\}_{j=1 \ldots N}$ and MRI volume M. Here, we represent image volumes in boldface $(\mathbf{H})$ and the corresponding slices with subscripts $\left(H_{j}\right)$. In the first part of each iteration, 3D registration is carried out on the current estimate of the histology volume and the MRI volume to obtain the transformation between the MRI axes to the specimen slicing axes. In the second part, 2D registration is carried out to obtain the transformations and deformations of each slide-mounted histology section using the current estimate of the aligned reference MRI. 


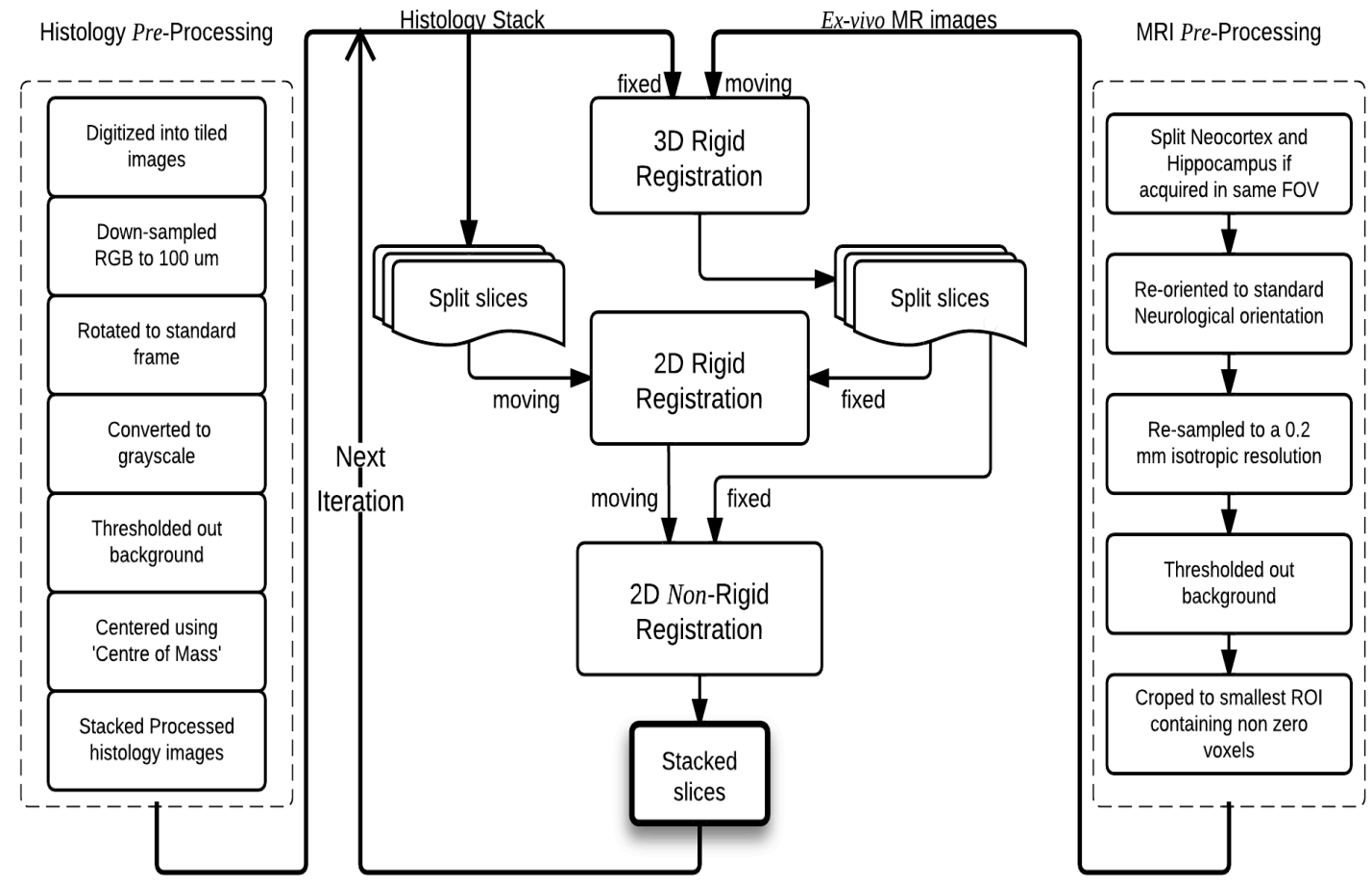

Figure 3: Registration pipeline showing pre-processing steps of the data and our iterative 2D-3D approach. The left column demonstrates the pre-processing steps applied to the histology slides sequentially from top to bottom. Likewise, the right column represents the pre-processing steps applied to the MRI of the specimens. The resulting histology stack acts as a fixed image to transform the MR image in a 3D rigid registration. The transformed image as well as the stack are split into slices $4.4 \mathrm{~mm}$ apart where each histology slice has a corresponding MRI slice. These MR slices act as fixed images to deform the histology slices rigidly then non-rigidly. The resulting deformed histology slices are stacked and fed back into the 3D rigid registration for the next iteration. 
Scan Setup II, containing both neocortex and hippocampus specimens in the same field of view, were converted from the scanner output Dicom $(\mathrm{dcm})$ format to the standard Nifti (nii) format, then bisected to produce separate volumes. Since the orientation of these specimens in the scanner bore did not correspond to the anatomical orientation, the orientation matrices of the images volumes were updated to reflect the correct pose. This operation was performed using photographs of the annotated specimens and 3D models of ex-vivo images, and the resulting orientation matrices were applied to all the acquired images in the session. The images were then background masked using a percentile threshold, resampled to $0.2 \mathrm{~mm}$ isotropic resolution, and cropped around the perimeter of the specimen.

\subsubsection{Histology image pre-processing}

The digitized histology images were similarly reoriented into a standard orientation, with the origin in the top-left image corner corresponding to superior-right in anatomical orientation, using the Aperio ImageScope software (Aperio Technologies, Vista, CA, USA) and the corresponding MRI as a reference. The images were then down-sampled to $100 \mu \mathrm{m}$ in-plane resolution and converted into NIFTI format, where each RGB channel was represented as a slice in a $3 \mathrm{D}$ volume. We converted the images to grayscale by extracting the green channel, since this channel was found to possess the best gray/white matter contrast in the H\&E stained slides. Finally, the images were background masked and centered in a standard $60 \mathrm{~mm}$ field of view using the image-based center-of-mass in each slide.

\subsubsection{Rigid registration}

Rigid registration in our iterative scheme was carried out with the flirt tool from (Jenkinson and Smith, 2001) (FSL, http://fsl.fmrib.ox.ac.uk/flirt) to perform the 3D and 2D registration. The default multi-modal cost function (correlation ratio) was applied and the registration was constrained to a rigid transform model with 6 and 3 degrees of freedom respectively for the 3D and 2D steps.

\subsubsection{Non-rigid registration}

A deformable registration between corresponding histology and MRI slices was employed to account for any anisotropic tissue deformations that can occur during histological processing, sectioning, and staining. We used a fast non-rigid registration that makes use of a B-spline deformation field and a normalized mutual information cost-function (Rueckert et al., 1999; Modat et al., 2010) (NiftyReg,http://sourceforge.net/ projects/niftyreg/). The B-spline image registration used a three-level multi-resolution image pyramid with final control point spacing of $2 \mathrm{~mm}$. Non-rigid registration was carried out starting at iteration 3 of the algorithm and not having been employed in the first two iterations to ensure the sufficient convergence of the rigid registration step. Furthermore, for slices where foreground of the MRI image or histology image were below a specified threshold, non-rigid registration was not performed and a zero deformation was assumed for the slice. The deformation penalty term (bending energy of the spline at a control point), was 
successively relaxed after each iteration to allow for greater deformations as the alignment is improved over each iteration. Specifically the sequence of bending energies employed at the iterative registration steps were $\{0.5,0.025,0.01\}$, for iterations $3-5$.

Due to histology tissue breakage and loss, a final registration step was added where binary 'ignore' masks defined on the registered histology slices were included in the deformable registration scheme. These ignore masks were manually-defined in 3D Slicer (http://www.slicer.org) using a large 2 mm radius paintbrush on regions of the MRI image where tissue loss is readily apparent in corresponding regions of the histology image, preventing these regions from contributing to the registration, which would result in incorrect deformations since one-to-one tissue correspondence is unattainable.

\subsection{Registration validation}

\subsubsection{Landmark-based validation}

To validate our registration protocol, we computed target registration error (TRE) based on manuallyidentified corresponding intrinsic landmarks on MR images and histology slices. These landmarks were used as independent targets to assess the accuracy of the registration at each iteration of the iterative registration scheme, as well as after deformable warping of the images.

We found that micro-vasculature or micro-bleeds that were visible on the H\&E histology slides appeared as dark hypo-intense regions in the ex-vivo T2-weighted MRI, as demonstrated in Figure 4. First, one rater identified landmarks on histology slides (downsampled to $10 \mu \mathrm{m}$ per pixel), restricting selection to vasculature with a transverse diameter of more than 35 pixels wide, assuming an ellipsoid shape. The most anterior and posterior histology slices in many cases of both specimens did not contain sufficient intact tissue for reliable placement of anatomical landmarks. Three raters then independently searched through the ex-vivo MRI to locate corresponding landmarks in the MRI images representing the centroid of these micro-vasculature or micro-bleeds. Since tissue contrast varies throughout the specimen, other MR scans of the specimen were used to facilitate the localization process. To compute the TRE the coordinates for all three raters were averaged to generate a consensus set of MRI landmarks. A total of 215 pairs of corresponding landmarks were identified for the TRE calculations in the twelve specimens. Note that only a single set of the histology landmarks were used to ensure consistent landmark locations.

\subsubsection{Localization error and statistical analysis}

Target localization incorporates human error in localizing the coordinates which combines with the image registration error to produce the TRE measurements. The target localization error (TLE) was calculated on ex-vivo MRI images as an unbiased estimator of the standard deviation of repeated localizations of the same landmark by the same rater (Fitzpatrick et al., 1998), described by the equation below: 


$$
T L E=\sqrt{\frac{1}{J} \sum_{j=1}^{J} \frac{1}{K-1} \sum_{K=1}^{K}\left\|P_{j, k}-\frac{1}{K} \sum_{k=1}^{K} P_{j, k}\right\|^{2}}
$$

where $P_{j, k}$ is the $k$-th localization of the $j$-th landmark. A total of five localization $(K=5)$ of twenty landmarks $(J=20)$ was performed.
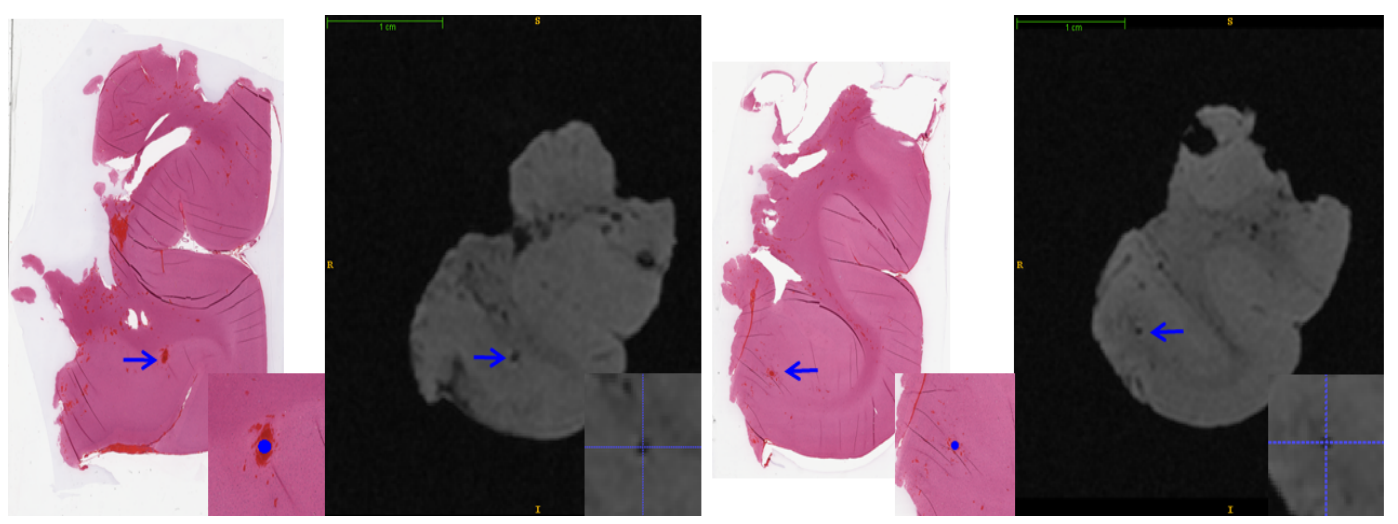

Figure 4: Preview of chosen intrinsic landmarks on histology and their localized corresponding landmark on Ex MRI. The arrows in blue pinpoint the micro vasculature used as targets for the TLE calculations on both histology and MRI. A zoomed in window demonstrates the targets on both modalities with cross hairs indicating the target coordinates on MRI and a circle showing the chosen coordinates on histology.

Inter-rater variability was measured as an estimator of the standard of deviation of repeated localization of the same landmarks by different raters, where a hundred and twenty eight landmarks $(J=128)$ were placed by three different raters $(K=3)$. Statistical analyses were performed in Prism 5.04 (GraphPad Software, San Diego, CA). To assess for significant differences between the several iterations of the algorithm and across rigid and non-rigid steps, we computed a repeated-measures analysis of variance (ANOVA) of the mean TRE value of each of these steps followed by Bonferroni multiple-comparison correction. A Bartlett's test for equal variances was conducted between all iterations (rigid and non-rigid) of the algorithm to verify the equal variance assumption of the ANOVA tests.

\section{Results}

The proposed methodology required $\sim 85$ min (including setup) for ex-vivo imaging and $<10$ min for execution of both the rigid and non rigid components of the algorithm (excluding the manual re-orientation step in the pre-processing scheme and the time for definition of ignore masks). Evaluation of the protocol was performed by localization of micro vasculature landmarks seen on both modalities by three raters. The localization protocol yielded 2-4 homologous landmarks on each of 8-12 sections per neocortex specimen and 1-3 landmarks on each of 6-8 sections per hippocampus specimen. Our registration protocol produced a 
mean target registration error of $0.76 \pm 0.66$ for hippocampal specimens, as shown in table 2 , and $0.98 \pm 0.60$ for neocortical specimens. The mean TRE was below $1.2 \mathrm{~mm}$ after the last step of the registration algorithm in all cases including both specimens. The mean landmark localization error for the three raters was found to be $0.21 \mathrm{~mm}$, relative to an MRI voxel size of $0.35 \times 0.35 \times 0.4 \mathrm{~mm}$. The inter-rater reliability between the raters was found to be $0.33 \mathrm{~mm}$. Figure 5 shows the registration errors across all steps of the algorithm including both rigid and non-rigid components for both specimens. The errors are shown first along iterations 1, 3 and 5 of the rigid component then the non-rigid steps beginning with a step using a high bending energy regularization penalty; then a low penalty weight and finally deformable registration utilizing ignore masks, that account for tissue breakage and differential shrinkage. The mean error of the rigid iterations reaches a plateau around iteration 5 for both specimens. The masked imaged based step outperformed the non-masked registration as expected by avoiding tissue breakage.

Figure 6 shows three neocortical slices with their corresponding MRI slices, that represents the location where the histology cuts were made with respect to the MRI scans of the resections, as well as the transformations of the slices after deformable registration. A picture of the resected specimen along with a volume rendering demonstrating the location of these histology slices in respect to the whole specimen are also shown in the figure. Checkerboard images of both rigid and non-rigid registration for a hippocampal slice are displayed in figure 7, which also shows a rendered representation of both sides of the hippocampus where the histology slice was cut.

The Bartlett's test confirmed the validity of the equal variance assumption for the ANOVA analyses of both specimens $(P>0.05)$. The significant results of the ANOVA analysis are shown in the 'Mean' row of tables 2 and 3. The ANOVA analysis, between the first deformable registration step (High Bending Energy) and the last rigid step (Iteration 5), failed to show a statistically significant difference of the mean TREs for both the hippocampus ( $P>0.05,95 \%$ Confidence Interval [CI] of difference -0.2666 to 1.653$)$ and neocortex $(P>0.05,95 \%$ CI -0.0147 to 1.54$)$. However, this test did demonstrate a significant decrease in TRE between (High Bending Energy) and the first rigid iteration (Iteration 1) for the hippocampus and neocortex respectively $(P \leq 0.01,95 \%$ CI 0.143 to 1.69 and $P \leq 0.05,95 \%$ CI -0.2040 to 1.715$)$. In comparison, decreasing the bending energy weight penalty produced significantly lower mean TRE than the final rigid iteration step (Iteration 5$)$ for the hippocampus and neocortex respectively $(P \leq 0.001,95 \%$ CI 0.449 to 2.00 and $P \leq 0.01,95 \%$ CI 0.3516 to 2.271). The proposed masked non-rigid scheme (Masked NR) had as well significantly lower TRE $(P \leq 0.0001)$ than Iteration5 for both the hippocampus $(95 \%$ CI 0.590 to $2.14 \mathrm{~mm}$ ) and neocortex (95\% CI $0.5143-2.434)$. 


\begin{tabular}{ccccccc}
\hline \hline & It.1 & It.3 & It.5 & H.B.E & L.B.E & Masked N.R \\
\hline Subj 1 & $2.07 \pm 0.96$ & $2.05 \pm 0.97$ & $2.05 \pm 0.98$ & $1.35 \pm 0.85$ & $0.88 \pm 0.67$ & $0.73 \pm 0.85$ \\
Subj 2 & $2.81 \pm 0.54$ & $2.87 \pm 0.63$ & $2.74 \pm 0.80$ & $2.52 \pm 0.94$ & $1.42 \pm 0.94$ & $1.03 \pm 0.94$ \\
Subj 3 & $2.13 \pm 1.78$ & $2.12 \pm 1.74$ & $2.09 \pm 1.73$ & $1.30 \pm 0.78$ & $0.73 \pm 0.42$ & $0.62 \pm 0.40$ \\
Subj 4 & $2.19 \pm 1.02$ & $2.09 \pm 1.02$ & $2.07 \pm 1.21$ & $1.01 \pm 0.54$ & $0.68 \pm 0.37$ & $0.68 \pm 0.42$ \\
Subj 5 & $2.05 \pm 0.84$ & $1.86 \pm 0.85$ & $1.78 \pm 0.95$ & $1.15 \pm 0.68$ & $0.75 \pm 0.43$ & $0.72 \pm 0.45$ \\
Mean & $2.25 \pm 1.10$ & $2.18 \pm 1.11$ & $2.15 \pm 1.14$ & $1.46 \pm 0.77^{\dagger}$ & $0.89 \pm 0.61^{\ddagger}$ & $0.76 \pm 0.66^{\ddagger}$ \\
\hline \hline
\end{tabular}

Table 2: TRE values for hippocampal registration across iterations. H.B.E: Non-rigid with a High Bending Energy penalty, L.B.E: Non-rigid with a Low Bending Energy penalty. $\dagger: P \leq 0.01$ between means registration step and Rigid Iteration $1 . \ddagger: P \leq 0.01$ between means of registration step and Rigid Iteration 5 .

\begin{tabular}{ccccccc}
\hline \hline & It.1 & It.3 & It.5 & H.B.E & L.B.E & Masked N.R \\
\hline Subj 1 & $2.97 \pm 0.86$ & $2.98 \pm 0.86$ & $2.96 \pm 0.87$ & $1.55 \pm 1.21$ & $1.26 \pm 0.66$ & $1.08 \pm 0.52$ \\
Subj 2 & $2.36 \pm 0.83$ & $1.92 \pm 0.84$ & $1.91 \pm 0.94$ & $1.69 \pm 1.17$ & $0.83 \pm 0.85$ & $0.72 \pm 0.68$ \\
Subj 3 & $2.23 \pm 1.40$ & $2.08 \pm 1.37$ & $2.07 \pm 1.37$ & $1.15 \pm 1.15$ & $0.98 \pm 0.99$ & $0.83 \pm 0.70$ \\
Subj 4 & $2.41 \pm 1.61$ & $2.40 \pm 1.76$ & $2.38 \pm 1.89$ & $2.17 \pm 0.88$ & $1.17 \pm 0.68$ & $1.05 \pm 0.65$ \\
Subj 5 & $2.19 \pm 1.02$ & $2.08 \pm 1.02$ & $2.06 \pm 1.01$ & $1.01 \pm 0.43$ & $1.02 \pm 0.45$ & $0.87 \pm 0.38$ \\
Subj 6 & $2.23 \pm 1.63$ & $2.24 \pm 1.65$ & $2.25 \pm 1.65$ & $2.06 \pm 0.61$ & $1.35 \pm 0.57$ & $1.12 \pm 0.52$ \\
Subj 7 & $2.16 \pm 1.41$ & $2.14 \pm 1.44$ & $2.11 \pm 1.44$ & $1.42 \pm 0.55$ & $1.15 \pm 0.49$ & $1.15 \pm 0.35$ \\
Mean & $2.37 \pm 1.19$ & $2.26 \pm 1.22$ & $2.25 \pm 1.28$ & $1.60 \pm 1.01^{\dagger}$ & $1.11 \pm 0.75^{\ddagger}$ & $0.98 \pm 0.60^{\ddagger}$ \\
\hline \hline
\end{tabular}

Table 3: TRE values for neocortical registration across iterations.H.BE: Non-rigid with a High Bending Energy penalty,L. B.E: Non-rigid with a Low Bending Energy penalty. $\dagger: P \leq 0.01$ between means registration step and Rigid Iteration $1 . \ddagger: P \leq 0.01$ between means of registration step and Rigid Iteration 5. 
Hippocampus

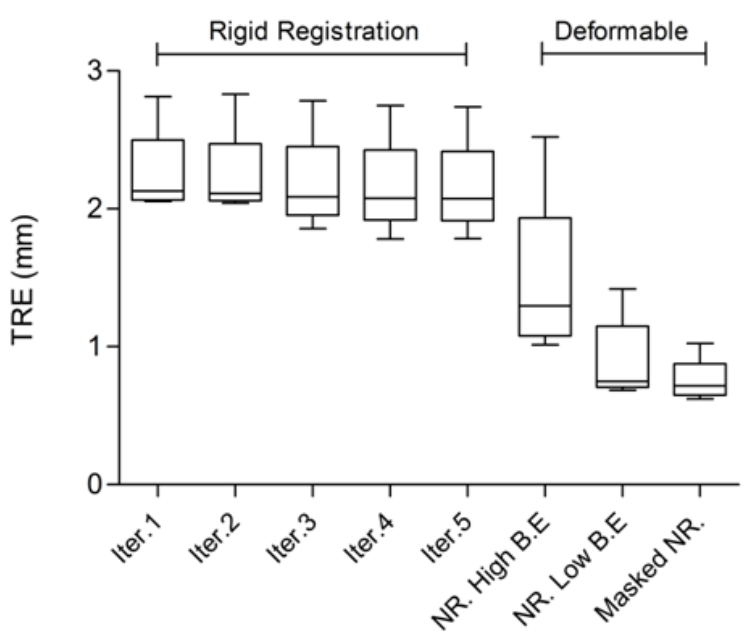

Neocortex

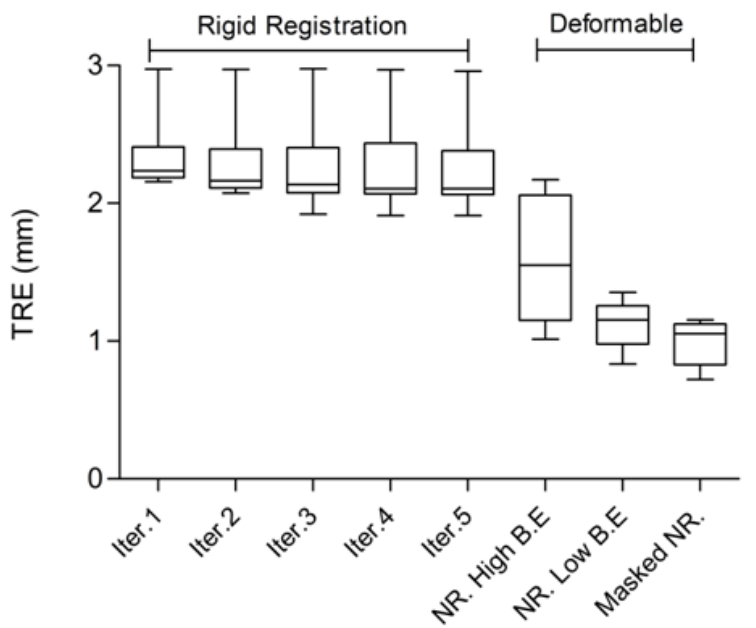

Figure 5: Boxplots with 5-95\% whiskers of Hippocampal and Neocortical registration target registration errors at each stage of the iterative registration scheme.

\section{Discussion}

In this article, we have described a method to reliably register ex-vivo MRI and sparsely sliced histology slides of neocortex and hippocampus specimens. Our protocol is a landmark free algorithm that produced sub-millimeter accuracy for hippocampal registration and close to 1-mm of error for temporal lobe neocortical registration. Correlating MRI with histopathology is imperative in the validation of new imaging sequences, since verification of pathological anomalies underlying signal changes is needed to enable these sequences to ultimately gain clinical acceptance. The intrinsic higher resolution of ex-vivo MR images provides a superior opportunity to further examine the correlation between MRI and histology. By addressing many challenges of the in-vivo MRI to histology registration, our protocol leaves single modality registration between specimen and preoperative MRI scans as the remaining step. In addition, ex-vivo to MRI registration can be used to validate specimen imaging, as it has been shown by Madabhushi et al. (2005) in prostate ex-vivo imaging examples. While using an intermediate ex vivo registration interrupts the clinical flow for specimen imaging, our algorithm requires $\sim 9$ min \pm 37 seconds on average (for the automated iterative $3 \mathrm{D} / 2 \mathrm{D}$ rigid approach plus the non-rigid steps) to register $100 \mu \mathrm{m}$ coronally sliced (anterior to posterior) histology slices to ex-vivo MRI images.

Previous studies on registration of histopathology to in-vivo imaging were reported mostly for rodents (Jacobs et al., 1999; Humm et al., 2003; Meyer et al., 2006; Lebenberg et al., 2010) and primates (Malandain 
a)

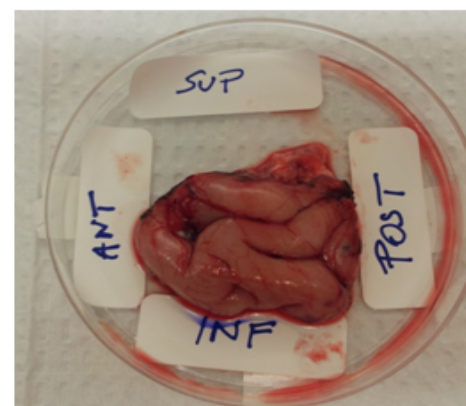

b)

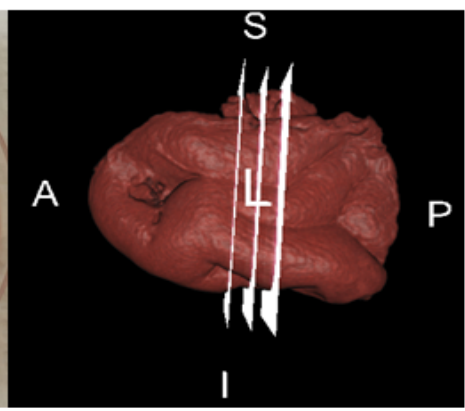

c)

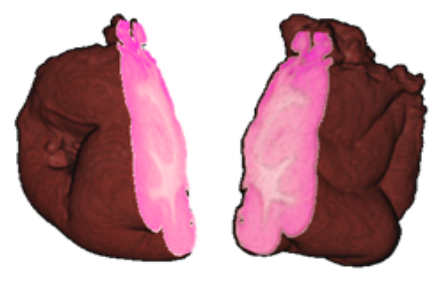

d)

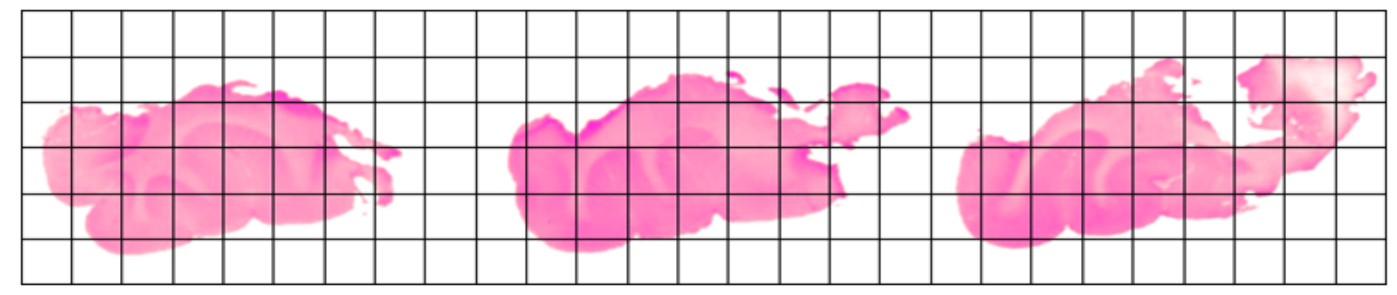

e)

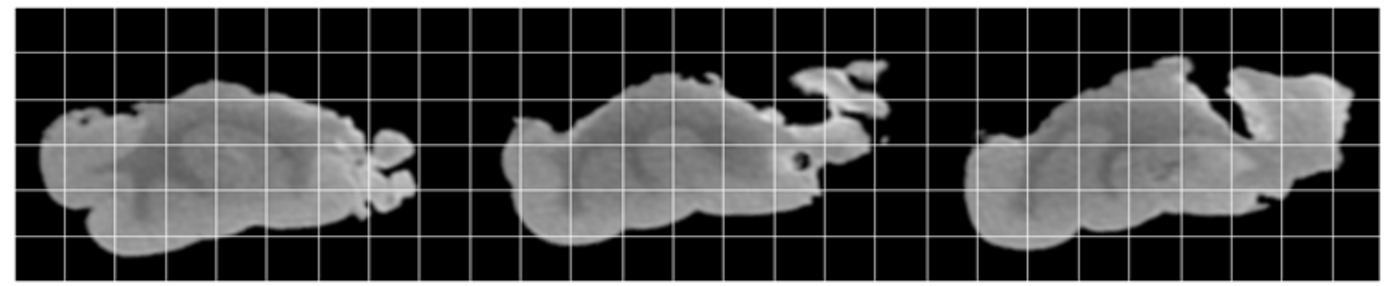

f)

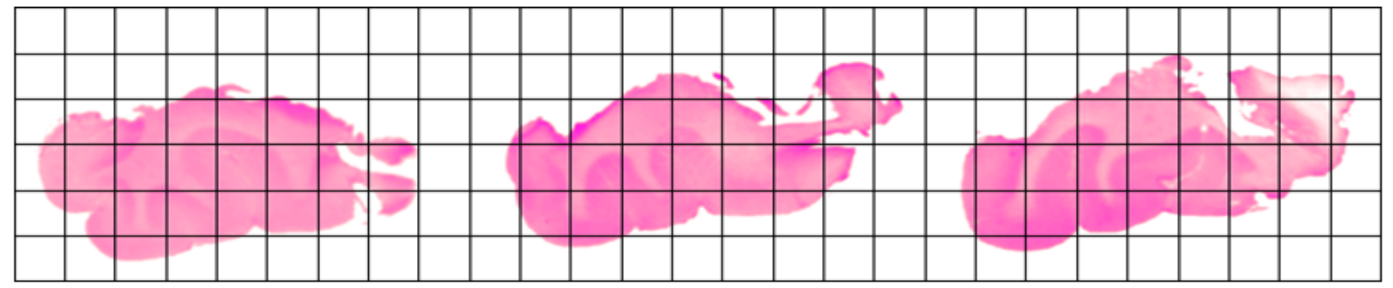

Figure 6: Example of a neocortex rigid and deformable registration showing: a) photograph of a neocortical specimen after resected with orientation labels placed by the operating surgeon, b) volume rendering of the MRI of the specimen showing the location of three consecutive histology slices. c) rendering of both sides of the specimen where the middle slice of histology was cut, d) the three histology slices shown in b), e) the corresponding MRI slices after 3D rigid registration, and f) the deformed histology slices after non-rigid registration to their corresponding MRI slices. 
a)

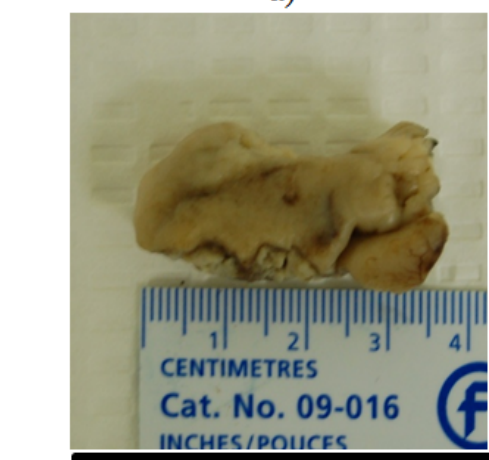

b)

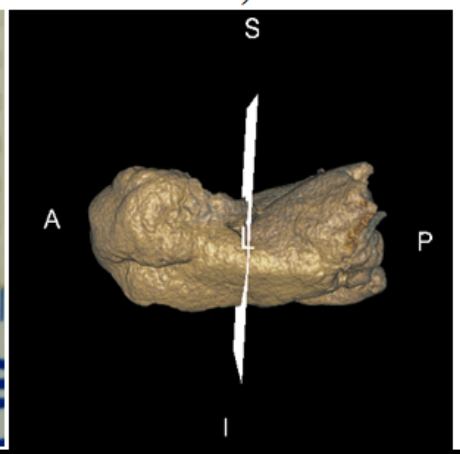

c)

d)

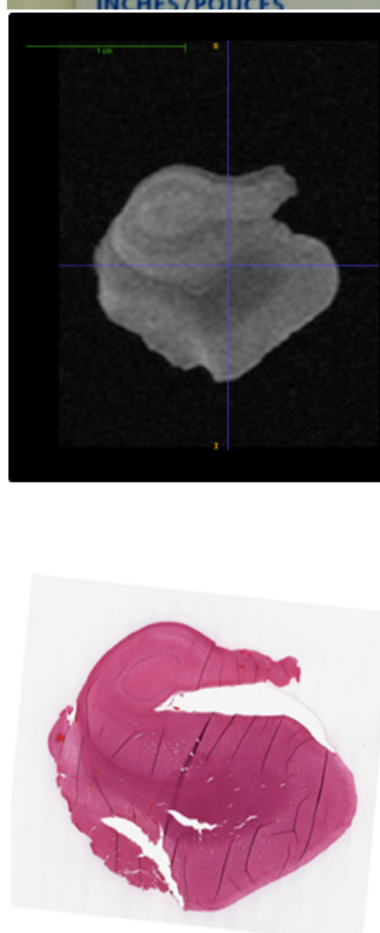

e)

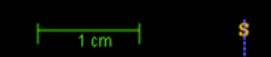

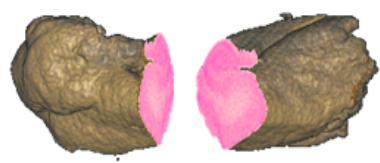
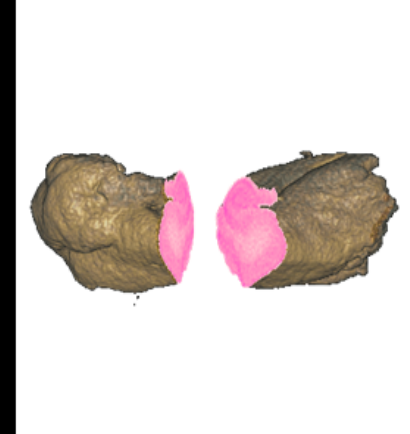

(1)
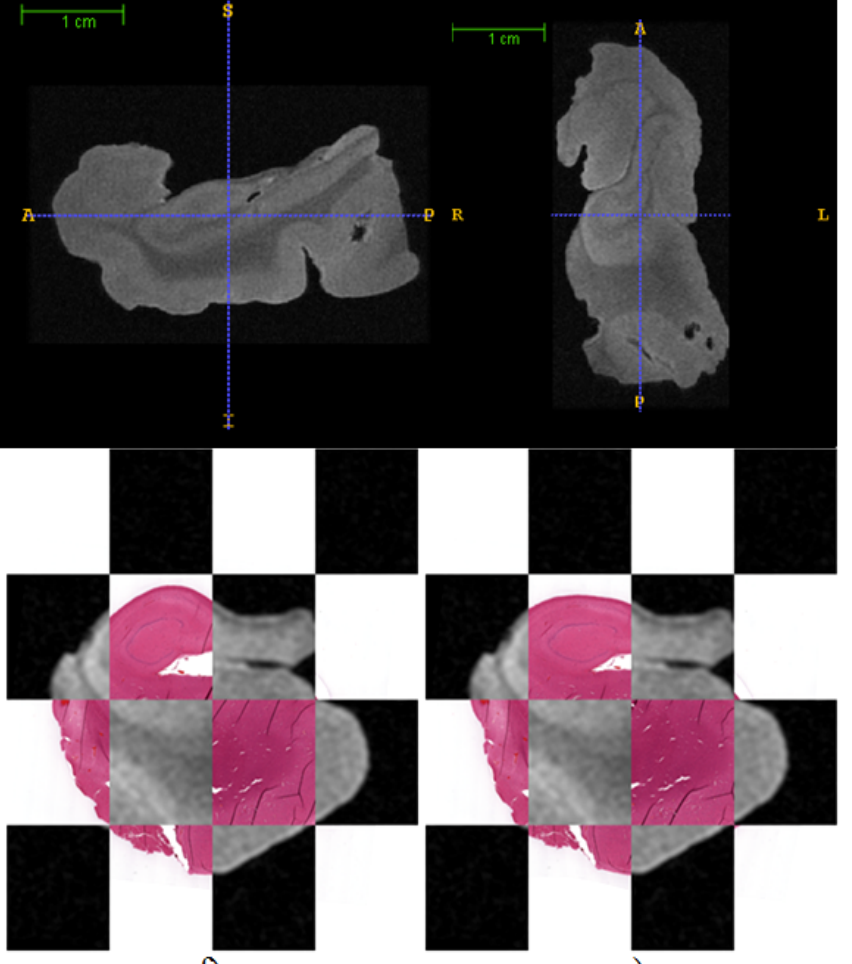

f)

g)

Figure 7: Example of a hippocampal rigid and deformable registration showing: a) photograph of a hippocampus before grossing, b) volume rendering of the MRI of the hippocampus demonstrating the location of a histology slice through the specimen, c) rendering of both sides of the hippocampus where the histology slice was cut, and d) three orthogonal views of the hippocampal MRI (left to right: coronal, sagittal, axial). The bottom row depicts: e) a coronal view of the same histological slice, f) a checkerboard image showing the MRI and histology before non-rigid registration, and g) a checkerboard image showing the MRI and non-rigidly deformed histology slice. 
et al., 2004; Breen et al., 2005; Dauguet et al., 2007; Ceritoglu et al., 2010). Relatively few studies were developed to register human whole-brain or single hemisphere postmortem MRI with histology (Schormann et al., 1995; Kim et al., 2000; Singh et al., 2008). These landmark-based and image-based registration algorithms, however, are not likely to be applicable to the registration of specimens from lobectomies to full preoperative MR images, due to the drastic change in shape and coherence when the specimen is separated from neighbouring tissue. Several previous works reconstructed a 3D histology volume from serially sectioned brain specimens at $<700 \mu \mathrm{m}$ to register to the MRI volume (Bardinet et al., 2002; Humm et al., 2003; Dauguet et al., 2007; Lebenberg et al., 2010; Chakravarty et al., 2006). This technique while producing accurate results is not compatible with the clinical work flow of pathology departments, where the tissue is sparsely sectioned at a thickness of more than a few mm. Other methods have been proposed that allow co-registration of histology to other modalities through the use of stereotactic systems using target points (Schmierer et al., 2003; Humm et al., 2003), however the design of these systems is tissue-specific and is not broadly applicable to other brain resections.

The measurement of TRE requires the identification of homologous landmarks on images of both modalities used for registration and is frequently lacking in analyses of these methods. A few articles have quantified and reported TRE in brain histology to MRI. Jacobs et al. (1999) reported a registration residual root-mean square (RMS) error of $0.83 \mathrm{~mm}$ between histological sections and MRI of ischemic rats and Humm et al. (2003) obtained a $0.25 \mathrm{~mm}$ registration error for tumor xenografts of one mice using stereotactic fiduciary markers. Both studies could not be extrapolated or compared to human cases due to the methodological differences between these protocols and resected human specimens, as well as the mechanical differences between excised human and whole primate brain. Singh et al. (2008) reported a $5.1 \mathrm{~mm}$ TRE computed as $3 \mathrm{D}$ coordinates of centroid of marked lesions in both modalities, which exceeds the desired error range for correlation between histopathology and MRI in focal epilepsy as underlying pathologies may be found on the scale of millimetres. Our method produced a mean TRE of $0.76 \pm 0.66$ and $0.98 \pm 0.60$ for hippocampal and neocortical specimens respectively, which is sufficient for exploring underlying pathologies of focal epilepsy. For a very small FCD with a volume of $128 \mathrm{~mm}^{3}$ (Besson et al., 2008), the mean TRE obtained from our algorithm is able to achieve a $70 \%$ overlap of the FCD, assuming it is a sphere. Our mean TLE of $0.21 \mathrm{~mm}$ is indicative that the localization variability is not dominating in the TRE measurements. The significantly lower mean TRE for the latter deformable registration found by our ANOVA analysis motivates the use of the hierarchal bending energies as well as the incorporation of ignore masks.

A previous method proposed visual comparison of photographs of temporal lobe neoctorex tissue slices to MRI, and reported a $<2 \mathrm{~mm}$ difference between two observers in most cases (Eriksson et al., 2005). This manual matching technique suffers from a major limitation that is the lack of image registration between the histology and MRI, which in turn dictates the use of region of interest (ROI) based analysis in further studies of correlation (Eriksson et al., 2007, 2009; Lockwood-Estrin et al., 2012). This operator- 
based method incorporates human bias in locating the corresponding slice of MRI, which explains the 4-mm difference between raters in their last case. Only two ROIs in temporal lobe neocortical specimen were assessed in Lockwood-Estrin's and Eriksson's work to analyze histopathology to MRI correlation. In one instance a negative correlation was seen between grey matter T2 values of fast Flair (fFT2) and NeuN field fraction (Eriksson et al., 2007), and another no correlation was found between normalized FLAIR signal intensity (nFSI) and NeuN field fraction within these ROIs (Lockwood-Estrin et al., 2012). While the differences between fFT2 and nFSI may not necessarily explain this discrepancy, averaging across the whole area of the ROI may mask signal changes of pathologies smaller than the size of the ROI. Moreover, unlike our protocol, Eriksson et al. (2005) focused on matching temporal lobe neocortical specimen and no hippocampal correspondence was performed. Registration of the hippocampus is very challenging due to the smaller size of the resection and the higher susceptibility of the tissue to deform and the histology slices to break apart. Furthermore, performing an image-based registration allows exploratory hypothesisfree analysis at a voxel-wise level and does not require ROI definition, which may be more sensitive to subtle pathologies. Our protocol will be complemented with 3D in-vivo to ex-vivo MR registration in order to explore the correlations between MRI and histology at greater depth, and provide histopathological validation of multi-modal MRI analysis techniques.

\section{Conclusion}

We present here a protocol for registration of ex-vivo specimen MRI to histopathology, specifically hippocampal and neocortical temporal lobe sections. Sub-millimeter errors have been shown for ex-vivo MRI to histology registration on twelve collected specimens from seven patients. A successful registration between histology - currently considered as the ground truth - and post-operative MRI of resected tissue is imperative for better understanding of focal epilepsy at both the micro and macro levels. This correspondence is a key component towards achieving MRI and histology correlation by bringing together information from both domains.

\section{Acknowledgments}

The authors would like to thank Dr. Seyed Mirsattari, Dr. Robert Hammond and Dr. Andrew Parrent for their assistance and support throughout the study. This project is funded by the Canadian Institute of Health Research (CIHR) grant MOP 184807 and Canada Foundation for Innovation (CFI) grant 20994. MG is supported by the NSERC Create Grant CAMI award at Western University. AK is supported by the Canadian Institute of Health Research (CIHR) Fellowship. 


\section{References}

Bardinet, E., Ourselin, S., Dormont, D., Malandain, G., Tandé, D., Parain, K., Ayache, N., Yelnik, J., 2002. Co-registration of histological, optical and mr data of the human brain. Medical Image Computing and Computer-Assisted InterventionMICCAI 2002, 548-555.

Bernasconi, A., Bernasconi, N., Caramanos, Z., Reutens, D. C., Andermann, F., Dubeau, F., Tampieri, D., Pike, B. G., Arnold, D. L., 2000. T2 relaxometry can lateralize mesial temporal lobe epilepsy in patients with normal MRI. Neuroimage 12 (6), $739-46$.

Bernasconi, N., Duchesne, S., Janke, A., Lerch, J., Collins, D. L., Bernasconi, A., 2004. Whole-brain voxel-based statistical analysis of gray matter and white matter in temporal lobe epilepsy. Neuroimage 23 (2), 717-23.

Bernhardt, B. C., Worsley, K. J., Kim, H., Evans, A. C., Bernasconi, A., Bernasconi, N., 2009. Longitudinal and cross-sectional analysis of atrophy in pharmacoresistant temporal lobe epilepsy. Neurology 72 (20), 1747-54.

Besson, P., Andermann, F., Dubeau, F., Bernasconi, A., 2008. Small focal cortical dysplasia lesions are located at the bottom of a deep sulcus. Brain 131 (Pt 12), 3246-55.

Breen, M. S., Lancaster, T. L., Wilson, D. L., 2005. Correcting spatial distortion in histological images. Comput Med Imaging Graph 29 (6), 405-17.

Ceritoglu, C., Wang, L., Selemon, L. D., Csernansky, J. G., Miller, M. I., Ratnanather, J. T., 2010. Large Deformation Diffeomorphic Metric Mapping Registration of Reconstructed 3D Histological Section Images and in vivo MR Images. Front Hum Neurosci 4, 43.

Chakravarty, M. M., Bertrand, G., Hodge, C. P., Sadikot, A. F., Collins, D. L., 2006. The creation of a brain atlas for image guided neurosurgery using serial histological data. Neuroimage 30 (2), 359-76.

Dauguet, J., Delzescaux, T., Condé, F., Mangin, J.-F., Ayache, N., Hantraye, P., Frouin, V., 2007. Three-dimensional reconstruction of stained histological slices and 3D non-linear registration with in-vivo MRI for whole baboon brain. Journal of neuroscience methods 164 (1), 191-204.

de Tisi, J., Bell, G. S., Peacock, J. L., McEvoy, A. W., Harkness, W. F. J., Sander, J. W., Duncan, J. S., 2011. The long-term outcome of adult epilepsy surgery, patterns of seizure remission, and relapse: a cohort study. Lancet 378 (9800), $1388-95$.

Engel, J., 1998. Etiology as a risk factor for medically refractory epilepsy: a case for early surgical intervention. Neurology $51(5), 1243-4$.

Engel, J., Levesque, M. F., Shields, W. D., 1992. Surgical treatment of the epilepsies: presurgical evaluation. Clin Neurosurg $38,514-34$.

Eriksson, S. H., Free, S. L., Thom, M., Harkness, W., Sisodiya, S. M., Duncan, J. S., 2005. Reliable registration of preoperative MRI with histopathology after temporal lobe resections. Epilepsia 46 (10), 1646-53.

Eriksson, S. H., Free, S. L., Thom, M., Martinian, L., Symms, M. R., Salmenpera, T. M., McEvoy, A. W., Harkness, W., Duncan, J. S., Sisodiya, S. M., 2007. Correlation of quantitative MRI and neuropathology in epilepsy surgical resection specimens-T2 correlates with neuronal tissue in gray matter. Neuroimage 37 (1), 48-55.

Eriksson, S. H., Free, S. L., Thom, M., Symms, M. R., Martinian, L., Duncan, J. S., Sisodiya, S. M., 2009. Quantitative grey matter histological measures do not correlate with grey matter probability values from in vivo MRI in the temporal lobe. Journal of neuroscience methods 181 (1), 111-8.

Fish, D. R., Smith, S. J., Quesney, L. F., Andermann, F., Rasmussen, T., 1993. Surgical treatment of children with medically intractable frontal or temporal lobe epilepsy: results and highlights of 40 years' experience. Epilepsia 34 (2), $244-7$.

Fitzpatrick, J. M., West, J. B., Maurer, C. R., 1998. Predicting error in rigid-body point-based registration. IEEE Trans Med Imaging 17 (5), 694-702.

Gibson, E., Gómez, J., Moussa, M., Crukley, C., Bauman, G., Fenster, A., Ward, A., 2012. 3d reconstruction of prostate 
histology based on quantified tissue cutting and deformation parameters. In: Society of Photo-Optical Instrumentation Engineers (SPIE) Conference Series. Vol. 8317. p. 22

Goubran, M., Khan, A. R., Crukley, C., Buchanan, S., Santyr, B., deRibaupierre, S., Peters, T. M., 2012. Robust registration of sparsely sectioned histology to ex-vivo MRI of temporal lobe resections. Vol. 8314. p. 83141V.

Howe, K. L., Dimitri, D., Heyn, C., Kiehl, T.-R., Mikulis, D., Valiante, T., 2010. Histologically confirmed hippocampal structural features revealed by $3 \mathrm{~T}$ MR imaging: potential to increase diagnostic specificity of mesial temporal sclerosis. AJNR American journal of neuroradiology 31 (9), 1682-9.

Humm, J. L., Ballon, D., Hu, Y. C., Ruan, S., Chui, C., Tulipano, P. K., Erdi, A., Koutcher, J., Zakian, K., Urano, M., Zanzonico, P., Mattis, C., Dyke, J., Chen, Y., Harrington, P., O’Donoghue, J. A., Ling, C. C., 2003. A stereotactic method for the three-dimensional registration of multi-modality biologic images in animals: NMR, PET, histology, and autoradiography. Med Phys 30 (9), 2303-14

Jacobs, M. A., Windham, J. P., Soltanian-Zadeh, H., Peck, D. J., Knight, R. A., 1999. Registration and warping of magnetic resonance images to histological sections. Med Phys 26 (8), 1568-78.

Jenkinson, M., Smith, S., 2001. A global optimisation method for robust affine registration of brain images. Medical Image Analysis 5 (2), 143-56.

Kim, T., Singh, M., Sungkarat, W., Zarow, C., Chui, H., 2000. Automatic registration of postmortem brain slices to MRI reference volume. Nuclear Science, IEEE Transactions on 47 (4), 1607-1613.

Lebenberg, J., Hérard, A. S., Dubois, A., Dauguet, J., Frouin, V., Dhenain, M., Hantraye, P., Delzescaux, T., 2010. Validation of MRI-based 3D digital atlas registration with histological and autoradiographic volumes: An anatomofunctional transgenic mouse brain imaging study. Neuroimage 51 (3), 1037-1046.

Lockwood-Estrin, G., Thom, M., Focke, N. K., Symms, M. R., Martinian, L., Sisodiya, S. M., Duncan, J. S., Eriksson, S. H., 2012. Correlating 3T MRI and histopathology in patients undergoing epilepsy surgery. Journal of Neuroscience Methods 205 (1), 182-9.

Madabhushi, A., Feldman, M. D., Metaxas, D. N., Tomaszeweski, J., Chute, D., 2005. Automated detection of prostatic adenocarcinoma from high-resolution ex vivo MRI. IEEE Transactions on Medical Imaging 24 (12), $1611-25$.

Malandain, G., Bardinet, E., Nelissen, K., Vanduffel, W., 2004. Fusion of autoradiographs with an MR volume using 2-D and 3-D linear transformations. Neuroimage 23 (1), 111-27.

Meyer, C. R., Moffat, B. A., Kuszpit, K. K., Bland, P. L., Mckeever, P. E., Johnson, T. D., Chenevert, T. L., Rehemtulla, A., Ross, B. D., 2006. A methodology for registration of a histological slide and in vivo MRI volume based on optimizing mutual information. Mol Imaging 5 (1), 16-23.

Modat, M., Ridgway, G. R., Taylor, Z. A., Lehmann, M., Barnes, J., Hawkes, D. J., Fox, N. C., Ourselin, S., 2010. Fast free-form deformation using graphics processing units. Computer methods and programs in biomedicine 98 (3), $278-84$.

Rueckert, D., Sonoda, L. I., Hayes, C., Hill, D. L., Leach, M. O., Hawkes, D. J., 1999. Nonrigid registration using free-form deformations: application to breast MR images. IEEE Transactions on Medical Imaging 18 (8), $712-21$.

Schmierer, K., Scaravilli, F., Barker, G. J., Gordon, R., MacManus, D. G., Miller, D. H., 2003. Stereotactic co-registration of magnetic resonance imaging and histopathology in post-mortem multiple sclerosis brain. Neuropathol Appl Neurobiol 29 (6), $596-601$.

Schormann, T., Dabringhaus, A., Zilles, K., 1995. Statistics of deformations in histology and application to improved alignment with MRI. IEEE Transactions on Medical Imaging 14 (1), 25-35.

Singh, M., Rajagopalan, A., Kim, T.-S., Hwang, D., Chui, H., Zhang, X.-L., Lee, A.-Y., Zarow, C., 2008. Co-registration of In-Vivo Human MRI Brain Images to Postmortem Histological Microscopic Images. Int. J. Imaging Syst. Technol. 18 (5-6), 325-335.

Smith, S. J. M., 2005. EEG in the diagnosis, classification, and management of patients with epilepsy. Journal of Neurology, 
Neurosurgery \& Psychiatry 76 Suppl 2, ii2-7. 